\title{
Analysis of Processing Mechanism in Stealth Dicing of Ultra Thin Silicon Wafer
}

\author{
Etsuji OHMURA ${ }^{1}$, Masayoshi KUMAGAI ${ }^{2}$, Makoto NAKANO ${ }^{2}, \mathrm{Koji} \mathrm{KUNO}^{2}$, Kenshi FUKUMITASU \\ and Hideki MORITA ${ }^{2}$ \\ ${ }^{1}$ Div. Materials and Manufacturing Science, Osaka University, Japan, ohmura@mapse.eng.osaka-u.ac.jp \\ ${ }^{2}$ Hamamatsu Photonics K.K.
}

\begin{abstract}
:
In this study, "stealth dicing” (SD) was applied to ultra thin wafers $50 \mu \mathrm{m}$ in thickness. A coupling problem composed of focused laser propagation in single crystal silicon, along with laser absorption, temperature rise and heat conduction was analyzed by considering the temperature dependence of the absorption coefficient. When the depth of the focal plane is too shallow, the laser is also absorbed at the surface as the thermal shock wave reaches the surface. As a result, not only is an internal modified layer generated but ablation occurs at the surface as well. When the laser is focused at the surface, strong ablation occurs. Ablation at the surface is unfavorable because of the debris pollution and thermal effect on the device domain. It was concluded that there is a suitable depth for the focal plane so that the thermal shock wave propagates inside the wafer only. The optimum irradiating conditions such as pulse energy, pulse width, spot radius, and depth of focal plane can be estimated theoretically also for ultra thin wafer.
\end{abstract}

Keywords: laser materials processing, dicing, silicon wafer, internal processing, ultra thin wafer, thermal shock wave

\section{Introduction}

Blade dicing is generally used for the dicing of a silicon wafer. Because blade dicing is machining by a dicing saw, mechanical vibration and stress occur during processing, and unavoidable damage such as crack and chipping occur to a wafer. For example, the semiconductor wafers such as memories tend to become thinner and thinner. It is necessary to retard processing speed to decrease crack and chipping but it is unfavorable from a viewpoint of productivity. In addition, fatal contamination of the device is a problem because grinding fluid is used to retard the heat generation in the blade dicing.

A laser dicing method using laser ablation and laser breaking was developed to overcome such problems of blade dicing [1-3]. Because a laser beam is focused at the wafer surface in the laser dicing method, the laser energy is absorbed at the wafer surface. As a result, the wafer surface is ablated, or the deformation of the wafer surface is caused by the variation of the crystal structure. In the laser ablation method, the influence that heat and debris pollution generated in laser processing have on the device properties and reliability cannot be ignored. An initial crack is necessary in the laser breaking method, and crack propagation does not always follow the desirable line, and then straight line accuracy becomes problem.

A laser dicing method named "Stealth Dicing" (SD) was developed to solve such problems [4-6]. It is already being used in the manufacturing process of the device. The SD method has a feature that laser processing is performed in the inside of the wafer, which is quite different from the other laser dicing method. Because a laser wavelength which is permeable for silicon is used, the laser beam penetrates to the focal neighborhood inside the wafer. As a result, there is no damage at the wafer surface region where device is really formed. Also problems such as thermal effect to the device region or the debris pollution which are fatal in a conventional laser dicing technique can be solved. There are large advantages to this SD method. In this paper, heat conduction analysis was performed for the SD method when applied to a silicon wafer of $50 \mu \mathrm{m}$ thick, and the difference in the processing result depending on the depth of focus was investigated. Furthermore, the validity of the analytical result was confirmed by experiment.

2. General statement of stealth dicing and the formation mechanism of the inside modified layer

Figure 1 shows a schematic illustration of the formation of the inside modified layer by laser irradiation in the SD method. When a permeable nanosecond pulse

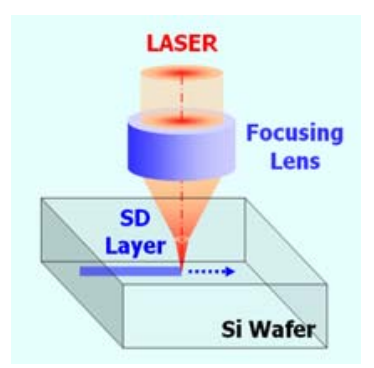

Figure 1 Schematic of stealth dicing 


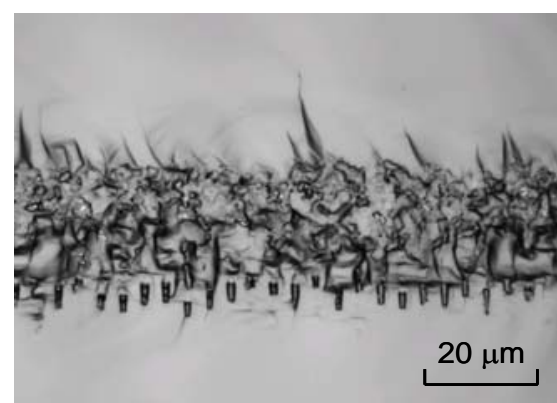

Figure 2 Internal modified layer generated in the inside of silicon wafer

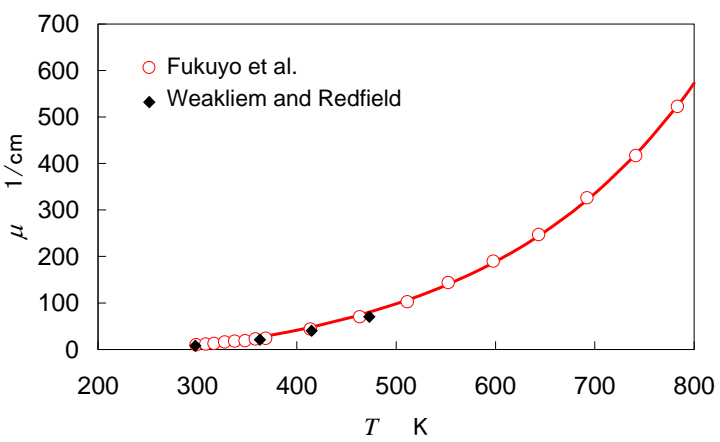

Figure 3 Temperature dependence of absorption coefficient of silicon single crystal for $1064 \mathrm{~nm}[10,11]$

laser is scanned in the horizontal direction with the focus on the inside of the silicon wafer, a belt-shaped high dislocation density layer is formed in a wafer. Cracks progress from the high dislocation density region to the monocrystal area. When a tensile stress is applied perpendicularly to this belt-shaped modified layer, the silicon wafer can be divided easily because cracks, which spread from the modified layer up and down, progress to the surface. The actual division in the SD processing is done by tape expansion. An example of the photographs of divided face of the SD processed silicon wafer is shown in Fig. 2. The central belt-shaped area is the modified layer. The pulsed laser was irradiated from the upside of the photograph and was scanned in the right direction. Voids are under the modified layer, and cracks are seen up and down.

Ohmura [7-9] conducted heat conduction analysis and simple thermal stress analysis by considering the temperature dependence of the absorption coefficient. Also, the formation mechanism of the inside modified layer was investigated. First of all, it was estimated that the 1,064nm laser, which penetrates silicon, was absorbed locally at the vicinity of the focal point due to the temperature dependence of absorption coefficient. Therefore the temperature dependence of absorption coefficient of the single crystal silicon was measured. The measurement result is shown in Fig. 3 [10, 11]. On the basis of this measurement result, the temperature distribution along the central axis of laser was calculated. As a result, the time variation of temperature was obtained and can be seen in Fig. 4. On the basis of these analysis results where the temperature dependence of absorption coefficient was considered, the following discussions were done: Laser absorption suddenly occurs in the focal

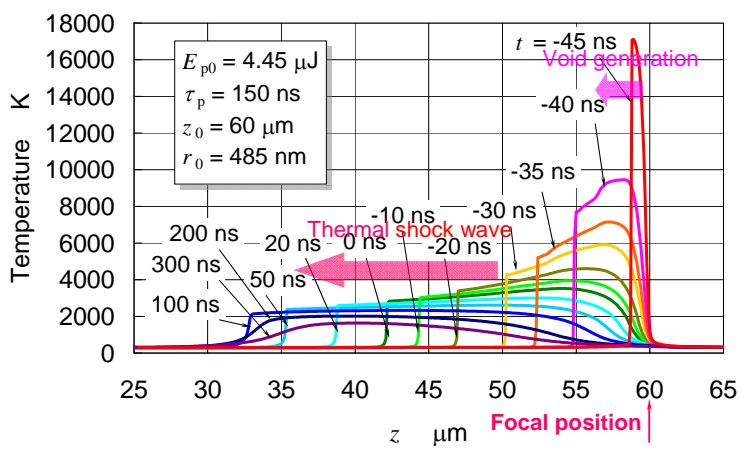

Figure 4 Time variation of temperature distribution along central axis

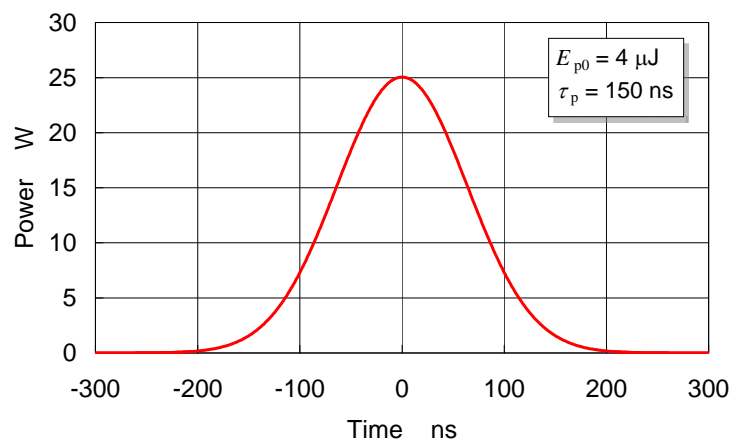

Figure 5 Pulse shape shown by the time variation of power

point vicinity, and a void is generated. Then a thermal shock wave propagates toward the upper surface, and the high dislocation density layer is formed. When the thermal shock wave pushes up the high dislocation density layer which occurred in the previous pulse irradiation, a crack, whose initiation is a dislocation, is produced. These discussions were able to explain the experimental results very well.

By the way, the 1,064nm laser was supposed in Fig. 4. The effective value of the pulse energy penetrating the silicon was $4.45 \mu \mathrm{J}$. The pulse shape was Gaussian. The pulse width of the half wave height full width value (FWHM) was $150 \mathrm{~ns}$, and $\mathrm{t}=0$ was considered to be the pulse center. The intensity distribution (spatial distribution) of the beam was also Gaussian. The thickness of the single crystal silicon was $100 \mu \mathrm{m}$, and the focal position was $60 \mu \mathrm{m}$ in depth. The initial temperature was $293 \mathrm{~K}$.

\section{Analysis method}

A $1,064 \mathrm{~nm}$ laser is considered here, and the internal temperature rise of $\mathrm{Si}$ by single pulse irradiation is analyzed. Considering that a laser beam is axisymmetric, we introduced the cylindrical coordinate system $\mathrm{O}-r \mathrm{Z}$ whose $z$-axis corresponds to the optical axis of laser beam and $r$-axis is taken on the surface of Si.

The absorption coefficient $\mu\left(T_{i, j}\right)$ in a lattice $(i, j)$ whose temperature is $T_{i, j}$ is expressed by $\mu_{i, j}$. When the Lambert law is applied between a small depth $\Delta z$ from depth $z=z_{j-1}$ to $z=z_{j}$, the laser intensity $I_{i, j}^{\prime}$ at the depth $z=z_{j}$ is expressed by $I_{i, j}^{\prime}=I_{i, j} e^{-\mu_{i, j} \Delta z}$, 
where $I_{i, j}$ is the laser intensity at the depth $z=z_{j-1}$.

The $1 / e^{2}$ radius at the depth $z$ of a laser beam which is focused with a lens is expressed by $r_{e}(z)$. In propagation of light waves from the depth $z=z_{j-1}$ to $z=z_{j}$, focusing or divergence of a beam can be evaluated by a parameter

$$
\gamma_{j}=r_{e}\left(z_{j}\right) / r_{e}\left(z_{j-1}\right) \text {, }
$$

The beam is focused when $\gamma_{j}$ is less than 1 , and is diverged when $\gamma_{j}$ is larger than 1 . Now, the laser intensity $I_{i, j}$ at the depth $z=z_{j-1}$ of a finite difference grid $(i, j)$ can be expressed by the energy conservation as follows:

(1) For $\gamma_{j-1}<1$

$$
I_{i, j}=\frac{\left(\gamma_{j-1}^{2} r_{i}^{2}-r_{i-1}^{2}\right) I_{i, j-1}^{\prime}+\left(1-\gamma_{j-1}^{2}\right) r_{i}^{2} I_{i+1, j-1}^{\prime}}{\gamma_{j-1}^{2}\left(r_{i}^{2}-r_{i-1}^{2}\right)},
$$

(2) For $\gamma_{j-1}>1$

$$
I_{i, j}=\frac{\left(\gamma_{j-1}^{2}-1\right) r_{i-1}^{2} I_{i-1, j-1}^{\prime}+\left(r_{i}^{2}-\gamma_{j-1}^{2} r_{i-1}^{2}\right) I_{i, j-1}^{\prime}}{\gamma_{j-1}^{2}\left(r_{i}^{2}-r_{i-1}^{2}\right)},
$$

Considering Eq. (1), the internal heat generation per unit time and unit volume in the grid $(i, j)$ is given by

$$
w_{i, j}=\left(1-e^{-\mu_{i, j} \Delta z}\right) I_{i, j} / \Delta z \text {, }
$$

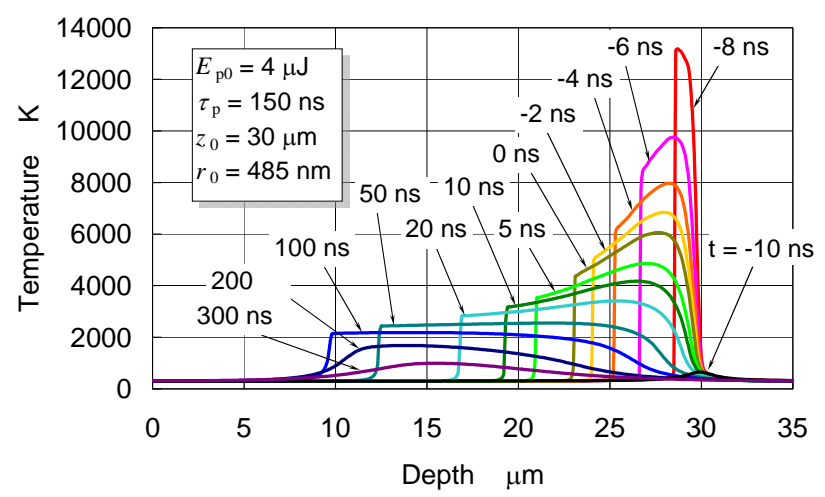

(a)

\section{Analysis results and discussions}

Concrete analyses were conducted under the irradiation conditions that the pulse energy, $E_{\mathrm{p} 0}$, was $4 \mu \mathrm{J}$, the pulse width (FWHM), $\tau_{\mathrm{p}}$, was $150 \mathrm{~ns}$, and the pulse shape was Gaussian. The pulse shape is shown in Fig. 5 with the power variation versus time. The pulse center was assumed to occur at $t=0$. The intensity distribution (spatial distribution) of the beam was assumed to be Gaussian. It was supposed that the thickness of single crystal silicon was $50 \mu \mathrm{m}$ and the depth of focal plane $z_{0}$ was $30 \mu \mathrm{m}, 15 \mu \mathrm{m}$ and $0 \mu \mathrm{m}$. The initial temperature was $293 \mathrm{~K}$.

The analysis region of silicon was a disk such that the radius was $111 \mu \mathrm{m}$ and the thickness was $50 \mu \mathrm{m}$. In the numerical calculation, the inside radius of $11 \mu \mathrm{m}$ was divided into 400 units at a width $25 \mathrm{~nm}$ evenly, and its outside region was divided into 622 units using a logarithmic grid. The thickness was divided into 10,000 units at $5 \mathrm{~nm}$ increments evenly in the depth direction. The time step was 10ps. The boundary condition was assumed to be a thermal radiation boundary.

\subsection{In the case of focal plane depth $30 \mu \mathrm{m}$}

The time variation of temperature at various depths along the central axis is shown in Fig. 6. Figure 6(b) shows the temperature distribution on a two-dimensional

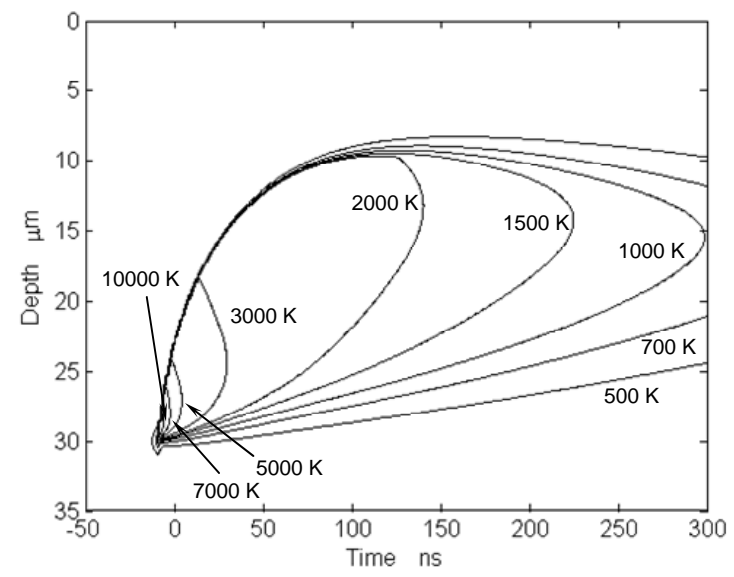

(b)

Figure 6 Time variation of temperature distribution along the central axis $\left(z_{0}=30 \mu \mathrm{m}\right)$

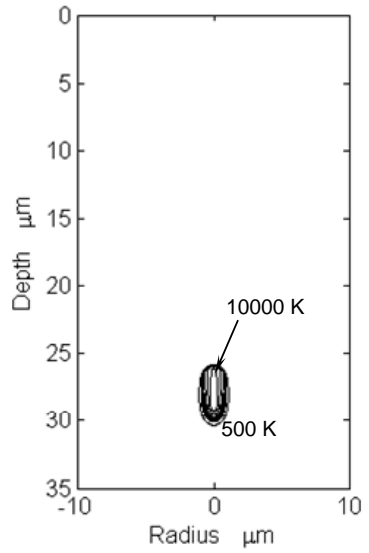

(a) $-5 \mathrm{~ns}$

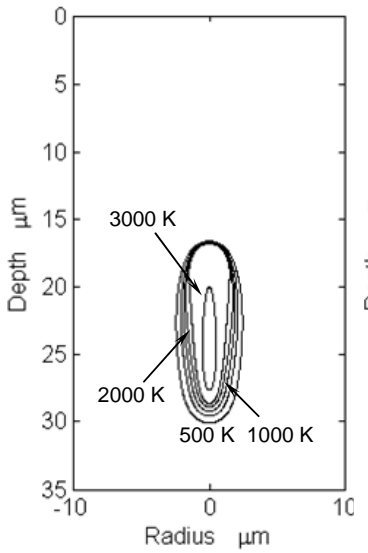

(b) $20 \mathrm{~ns}$

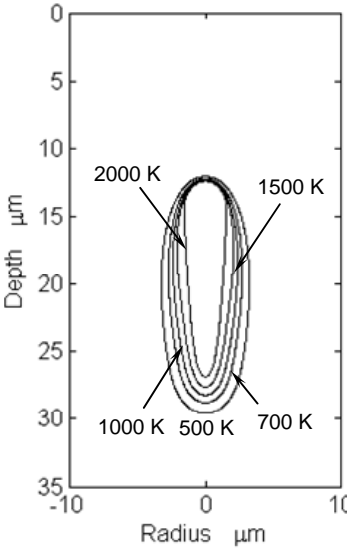

(c) $50 \mathrm{~ns}$

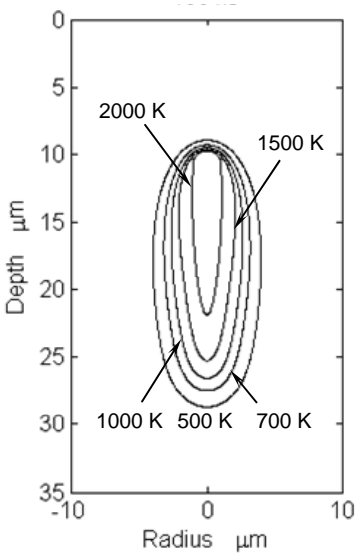

(d) $100 \mathrm{~ns}$

Figure 7 Time variation of temperature distribution $\left(z_{0}=30 \mu \mathrm{m}\right)$. Contour lines show 500, 700, 1000, 1500,2000, 3000, 5000, 7000 and $10000 \mathrm{~K}$. 
plane of depth and time by contour lines. It can be understood from Fig. 6(a) that laser absorption begins suddenly at a depth of $z=29 \mu \mathrm{m}$ at about $t=-8 \mathrm{~ns}$ and the temperature rises to about $12,000 \mathrm{~K}$ instantaneously. The region where the temperature rises beyond 8,000 K will be instantaneously vaporized and a void is formed. The high temperature area beyond 2,000 K then expands rapidly in the surface direction until $t=100 \mathrm{~ns}$ as shown in Fig. 6(b). The contour at the leading edge of this high temperature area is clear in this figure. Also the temperature gradient is steep as shown in Fig. 6(a). Therefore, this high-temperature area is named a thermal shock wave as well. It is calculated that the thermal shock wave travels at a mean speed of about $300 \mathrm{~m} / \mathrm{s}$. Propagation of the thermal shock wave is shown in Fig. 7 by a time variation of the two-dimensional temperature distribution. The contour of the high-temperature area is comparatively clear until $t=50 \mathrm{~ns}$, because the traveling speed of the thermal shock wave is much higher than the velocity of thermal diffusion. The contour of the high temperature area becomes gradually vague at $t=100 \mathrm{~ns}$ when the thermal shock wave propagation is finished. Because the temperature history is similar to the case of thickness $100 \mu \mathrm{m}$, the inside modified layer such as Fig. 2 is expected to be generated.

\subsection{In the case of focal plane depth $15 \mu \mathrm{m}$}

The time variation of the temperature distribution along the central axis in case of focal plane depth $15 \mu \mathrm{m}$ is shown in Fig. 8. It can be understood from Fig. 8(a) that laser absorption begins suddenly at a depth of $z=14 \mu \mathrm{m}$ at about $t=-10 \mathrm{~ns}$ and the temperature rises to about $12,000 \mathrm{~K}$ instantaneously. As well as the case of focal plane depth $30 \mu \mathrm{m}$, the region where the temperature rises beyond 8,000 $\mathrm{K}$ will be instantaneously vaporized and a void is formed. Then the thermal shock wave propagates in the surface direction until about $25 \mathrm{~ns}$. It is understood from Fig. 8(b) that laser absorption suddenly begins at the surface, once the thermal shock wave reaches the surface. Though the laser power already passes the peak, and gradually decreases (cf. Fig. 5), the surface temperature rises beyond $20000 \mathrm{~K}$, which is higher than the maximum temperature which is reached at the inside. Although the thermal diffusion velocity is fairly slower than the thermal shock wave velocity, the internal heat is diffused to the surrounding. However, because the heat in the neighborhood of the surface is diffused only in the inside of the lower half, the surface temperature becomes very high and is maintained comparatively for a long time.

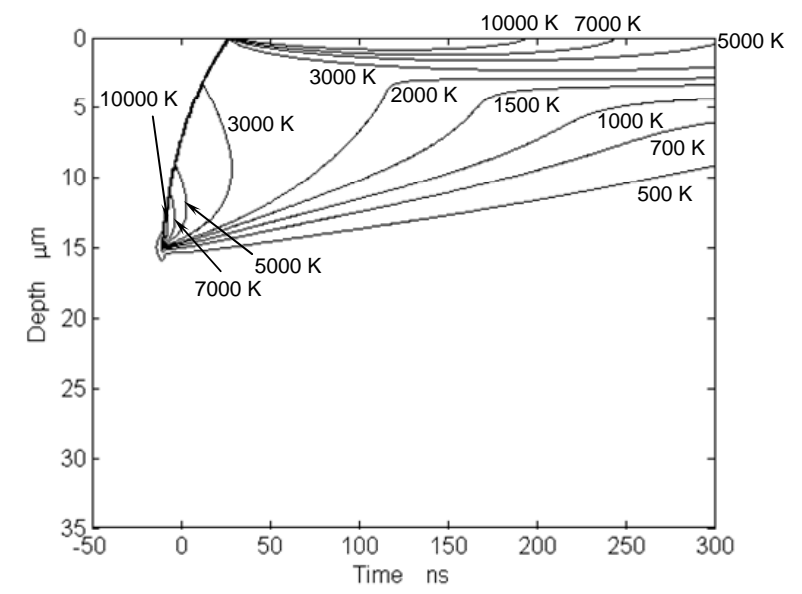

(b)

Figure 8 Time variation of temperature distribution along the central axis $\left(z_{0}=15 \mu \mathrm{m}\right)$

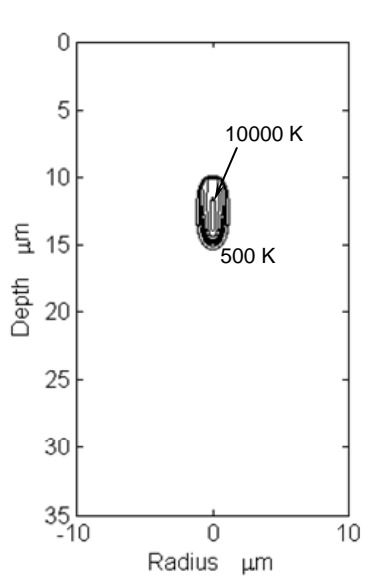

(a) $-5 \mathrm{~ns}$

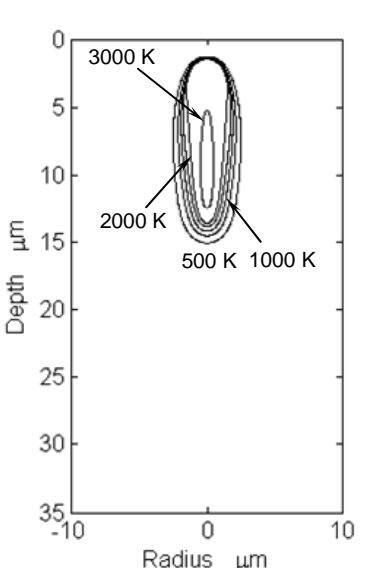

(b) $20 \mathrm{~ns}$

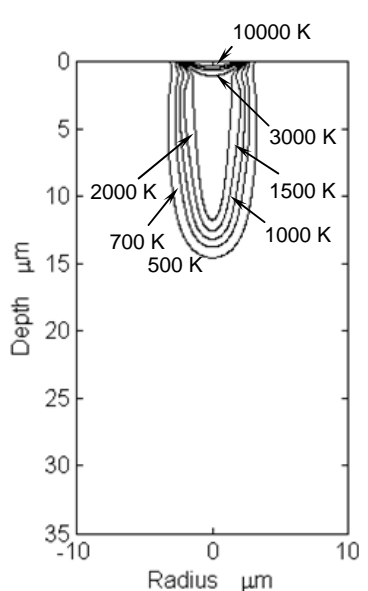

(c) $50 \mathrm{~ns}$

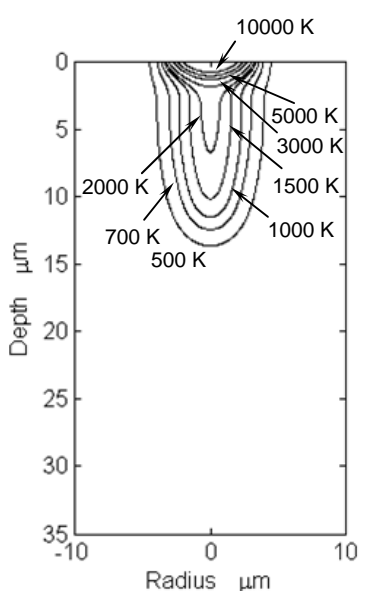

(d) $100 \mathrm{~ns}$

Figure 9 Time variation of temperature distribution $\left(z_{0}=15 \mu \mathrm{m}\right)$. Contour lines show 500, 700, 1000, 1500,2000, 3000, 5000, 7000 and $10000 \mathrm{~K}$. 

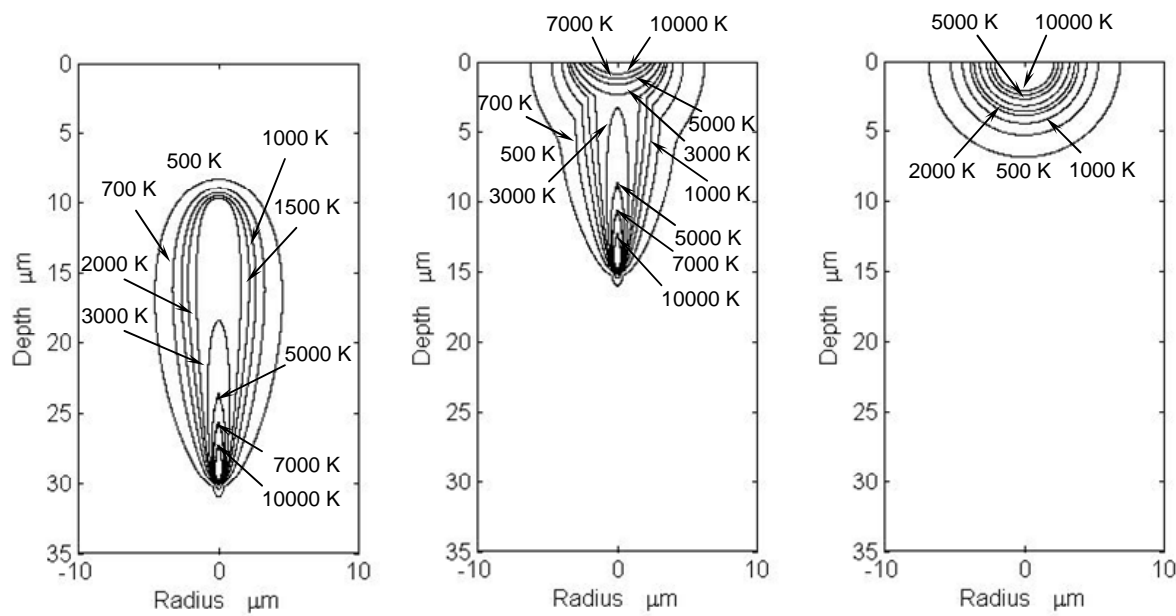

Figure 10 Comparison of the maximum temperature distribution. Contour lines show 500, 700, 1000, 1500,2000, 3000, 5000, 7000 and $10000 \mathrm{~K}$.

Ablation occurs of course in such a high-temperature state. As a result, it is expected that not only is an inside modified layer generated, but also the surface is removed by ablation. Figure 9 shows that the surface temperature rises suddenly after the thermal shock wave propagates in the inside of the silicon, reaches the surface, by the time variation of two dimensional temperature distribution.

\subsection{In the case of focal plane depth $0 \mu \mathrm{m}$}

When the laser is focused at the surface, laser absorption begins suddenly at the surface at $t=-35 \mathrm{~ns}$, and the maximum surface temperature in the calculation reaches $6 \times 10^{5} \mathrm{~K}$. It is estimated that violent ablation occurs when such an ultra-high temperature is reached. Because of the pollution of the device area by the scattering of the debris and thermal effect, the ablation at the surface is quite unfavorable.

\subsection{Comparison of the maximum temperature} distributions and the experimental results

The maximum temperature distributions at the focal plane depths of $30 \mu \mathrm{m}, 15 \mu \mathrm{m}$ and $0 \mu \mathrm{m}$ are shown in Fig.
10 in order to compare the previous analysis results at a glance. Because high-temperature area stays in the inside of the wafer when $z_{0}$ is $30 \mu \mathrm{m}$, it was estimated that the inside modified layer as shown in Fig. 2 will be generated. In the case of $z_{0}=15 \mu \mathrm{m}$, it was estimated that the surface is ablated although the modified layer is generated inside. In the case of $z_{0}=0 \mu \mathrm{m}$, it was estimated that the surface was ablated intensely. It is concluded from the above analysis results that the laser irradiation condition for SD processing should be selected at a suitable focal plane depth so that the thermal shock wave does not reach the surface.

In order to verify the validity of the estimated results, laser processing experiments were conducted under the same irradiation condition as the analysis condition. The repetition rate in the experiments was $80 \mathrm{kHz}$. The results are shown in Fig. 11. Optical microscope photographs of the top views of the laser-irradiated surfaces and the divided faces are shown in the middle row and the bottom row, respectively. Figure 11 (a), (b)

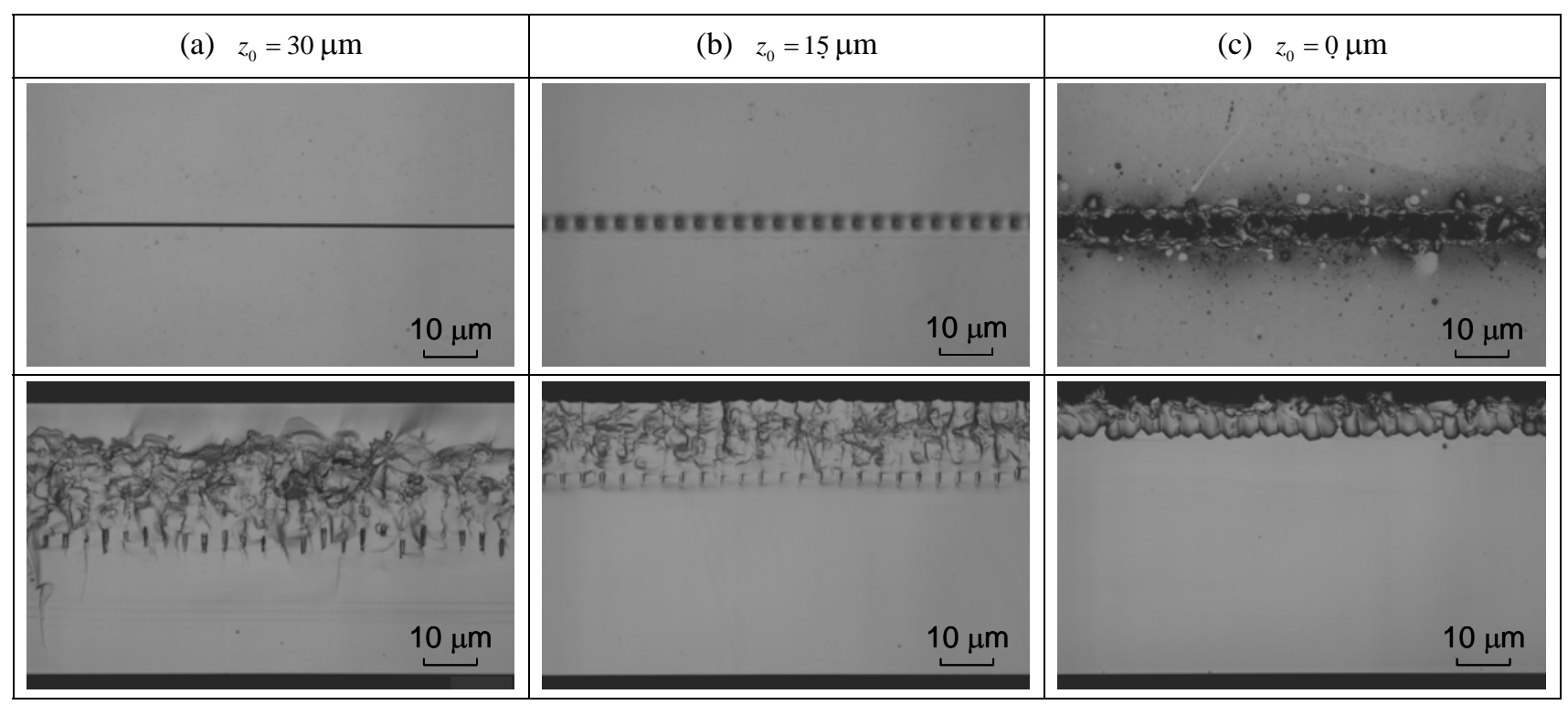

Figure 11 Experimental results $\left(E_{\mathrm{p}}=4 \mu \mathrm{J}, \tau_{\mathrm{p}}=150 \mathrm{~ns}, \quad v=300 \mathrm{~mm} / \mathrm{s}, \quad f_{\mathrm{p}}=80 \mathrm{kHz}\right)$ 
and (c) are results in the case of $z_{0}=30 \mu \mathrm{m}, z_{0}=15 \mu \mathrm{m}$, $z_{0}=0 \mu \mathrm{m}$, respectively. In the case of $z_{0}=30 \mu \mathrm{m}$ which is shown in Fig. 11 (a), it can be confirmed that voids are generated at the place that is slightly higher than the focal plane and the high dislocation density layer is generated in those upper parts, which are similar to Fig. 2. In the case of $z_{0}=15 \mu \mathrm{m}$ which is shown in Fig. 11 (b), it is recognized that voids are generated at the place that is slightly higher than the focal plane and the high dislocation density layer is generated in those upper parts. However, it is observed that the surface is ablated and holes are opened from the photograph of the laser irradiated surface. In the case of $z_{0}=0 \mu \mathrm{m}$ which is shown in Fig. 11 (c), it is seen that strong ablation occurs and debris is scattered to the surroundings. Voids and the high dislocation density layer are not recognized in the divided face. Only the cross section of the hole caused by ablation is seen. These experimental results agree fairly well with the estimation based on the previous analysis result. Therefore, the validity of the analytical model, the analysis method, and the analysis results of this study are proven. The processing results can be estimated to some extent by using the analysis model and the analysis method in the present study. It is useful in optimization of the laser irradiation condition.

\section{Conclusion}

In the stealth dicing (SD) method, the laser beam that is permeable for silicon is absorbed locally in the vicinity of the focal point, and an interior modified layer (SD layer), which consists of voids and high dislocation density layer, is formed. It was clarified by our previous analysis that the above formation was caused by the temperature dependence of the absorption coefficient and the propagation of a thermal shock wave. In this paper, the SD processing results of an ultra thin wafer of $50 \mu \mathrm{m}$ in thickness were estimated based on this analytical model and analysis method. Particularly we paid attention to the difference in the results depending on the focal plane depth. Furthermore, in order to compare with the analysis results, laser processing experiments were conducted with the same irradiation condition as the analysis conditions.

In the case of focal plane depth $z_{0}=30 \mu \mathrm{m}$, the analysis result of temperature history was similar to the case when the wafer thickness is $100 \mu \mathrm{m}$ and the focal plane depth is $60 \mu \mathrm{m}$. Therefore, it was predicted that a similar inside modified layer will be generated. In the case of $z_{0}=15 \mu \mathrm{m}$, it was estimated that not only the inside modified layer is generated, but also the surface is ablated. Because the thermal shock wave reached the surface, remarkable laser absorption occurred at the surface. In the case of $z_{0}=0 \mu \mathrm{m}$, it was estimated that the surface is ablated intensely. These estimation results agreed well with experimental results. Therefore, the validity of the analytical model, the analysis method and the analysis results of this study was proven.

As conclusion of this paper, the following points became clear:
(1) When the analytical model and the analysis method of the present study are used, the processing mechanism can be understood well, and the processing results can be estimated to some extent. It is useful in optimization of the laser irradiation condition.

(2) There is a suitable focal plane depth in the SD processing, and it is necessary to select the laser irradiation condition so that the thermal shock wave does not reach the surface.

\section{References}

[1] G.C. Lim, A.T. Mai, D. Low and Q. Chen: High Quality Laser Microcut-ting of Difficult-to-Cut Materials - Copper and Si Wafer, Proceedings of the 21st International Congress on Applications of Lasers and Electro-Optics, (2002).

[2] R. Ebutt, S. Danyluk and I. Weisshaus: Method to Evaluate Damage Induced by Dicing and Laser Cut-ting of Si Wafers, Microstructural Science, 23, (1996) 89.

[3] T. Ueda, K. Yamada, K. Oiso and A. Hosokawa: Thermal Stress Cleaving of Brittle Materials by Laser Beam, Annals of CIRP, 51/1, (2002) 149.

[4] Japanese Patent 3408805 and U.S.A. Patent 6992026 etc.

[5] F. Fukuyo, K. Fukumitsu and N. Uchiyama: The Stealth Dicing Technologies and Their Application, Proceedings of the 6th Laser Precision Micro-fabrication, (2005).

[6] K. Fukumitsu, M. Kumagai, E. Ohmura, H. Morita, K. Atsumi and N. Uchiyama: The Mechanism of Semiconductor Wafer Dicing by Stealth Dicing Technology, Proceedings of the 4th International. Congress on Laser Advanced Materials Processing, (2006).

[7] E. Ohmura, F. Fukuyo, K. Fukumitsu and H. Morita, Internal Modi-fied-layer Formation Mechanism into Silicon with Nanosecond Laser, Journal of Achievements in Materials and Manufacturing Engineering, 17, (2006), 381.

[8] E. Ohmura, K. Fukumitsu, N. Uchiyama, K. Atsumi, M. Kumagai and H. Morita: Analysis of Internal Modified-Layer Formation Mechanism in Stealth Dicing, Proceedings of the 66th Laser Materials Processing Conference, (2006), 19. (in Japanese)

[9] E. Ohmura, K. Fukumitsu, N. Uchiyama, K. Atsumi, M. Kumagai and H. Morita: Analysis of Modified Layer Formation into Silicon Wafer by Per-meable Nanosecond Laser, Proceedings of the 25th International Congress on Application of Laser and Electro-Optics 2006 (ICALEO2006), Laser Microfabrication Conference, CD-ROM, (2006), 24.

[10] F. Fukuyo, E. Ohmura, K. Fukumitsu and H. Morita: Measurement of Temperature Depend-ence of Absorption Coefficient of Single Crystal Silicon, Journal of Japan Laser Processing Society, 14-1, (2007), 22. (in Japanese)

[11] Weakliem, H.A. and Redfield, D.: Temperature Dependence of the Optical Properties of Silicon, Journal of Applied Physics, 50-3, (1979), 1491. 